\title{
Etio-Hematological Profile and Clinical Correlates of Outcome of Pancytopenia in Children: Experience From a Tertiary Care Center in North India
}

\author{
Muniba Alim ${ }^{1,2,3}$, Nishant Verma ${ }^{1}$, Archana Kumar ${ }^{4,1}$, Vishal Pooniya ${ }^{1}$, Rafey Abdul Rahman ${ }^{5}$ \\ 1. Pediatrics, King George's Medical University, Lucknow, IND 2. Clinical Hematology, Sanjay Gandhi Postgraduate \\ Institute of Medical Sciences, Lucknow, IND 3. Pediatrics, Uttar Pradesh University of Medical Sciences, Etawah, IND 4 \\ Pediatrics, Era's Lucknow Medical College and Hospital, Lucknow, IND 5. Pediatric Surgery, Uttar Pradesh University of \\ Medical Sciences, Etawah, IND
}

Corresponding author: Muniba Alim, munibaalim@gmail.com

\begin{abstract}
Introduction

Pancytopenia is a clinical entity encountered in pediatric practice as a feature of various benign and malignant disorders. It describes the simultaneous presence of anemia, leucopenia and thrombocytopenia. Attempts to identify the correct etiology and gauging the severity of pancytopenia will help to determine the management and prognosis of the patients.
\end{abstract}

\section{Objectives}

To study etio-hematological profile, clinical correlates and outcome of pancytopenia in Indian children

\section{Methods}

This prospective observational study of children with pancytopenia was conducted at a tertiary care center from August 2015 to July 2016. Clinical, hematological and bone marrow studies were performed and patients were followed for one year. The collected data were statistically analyzed.

\section{Results}

Out of 84 cases, the mean age at diagnosis was 70 (70.77 \pm 4.8$)$ months. Bone marrows showed aplastic changes in $37 \%$ and hyperplasia in $14 \%$ of patients. In our study, most common causes were aplastic anemia, acute leukemia and nutritional anemia. During the first year of follow-up, 67\% pancytopenics survived and $12 \%$ succumbed (rest discontinued treatment) with $~ 26 \%$ of aplastic anemia ( $7 / 27$ cases) and $9 \%$ of acute leukemia ( $2 / 22$ cases) not surviving. Anthropometric status of patients and severity in aplastic anemia were significantly associated $(\mathrm{p}<0.05)$ with outcome.

Review began 04/19/2021 Review ended 05/26/2021 Published 06/02/2021

\section{(c) Copyright 2021}

Alim et al. This is an open access article distributed under the terms of the Creative Commons Attribution License CC-BY 4.0., which permits unrestricted use, distribution, and reproduction in any medium, provided the original author and source are credited.

\section{Conclusion}

The data gathered support a complex picture for pancytopenia in our study population since both benign nutritional deficiencies and malignant hematological neoplasms were common. Bone marrow studies seem to be of salient use in delineating etiology. As the outcome is multifactorial, factors like anthropometry, hematological parameters have a bearing on prognosis.

Categories: Pediatrics, Epidemiology/Public Health, Hematology

Keywords: aplastic anemia, hematology, pancytopenia, pediatrics, etiology

\section{Introduction}

Pancytopenia is the decrease in all three cellular elements of peripheral blood, that is, red blood cells, white blood cells and platelets leading to anemia, leukopenia and thrombocytopenia. It is encountered in clinical practice as a spectrum of multiple diseases, ranging from non-malignant conditions including drug-induced, infections or nutritional deficiencies to malignant neoplasms including primary hematological or nonhematological metastatic malignancies. Considering that both treatment and prognosis depend on the cause of pancytopenia, it is essential to reach a definitive diagnosis early [1,2]. The studies in this field have summarised that the varied causes of pancytopenia can be attributed to the geographic area, genetic differences, stringency of diagnostic criteria, and differences in the methodology used. There are varying trends in its clinical pattern, treatment modalities, and outcomes that are not only appreciated in different countries but also in different regions of a single country. From North India, there have been few studies in this area. So the present study has been undertaken to evaluate the various etiologies, clinico-hematological 


\section{Materials And Methods Study settings}

This study was conducted in a medical university, a tertiary level care center located in Uttar Pradesh, India which caters to millions of people of North India. It has a well-established department of Pediatrics which is one of the best departments in India for managing pediatric diseases including hematological disorders. The Department of Pediatrics at this University recruits children from Uttar Pradesh and adjoining states of Bihar, Uttarakhand, Madhya Pradesh, Chhattisgarh and parts of Nepal.

\section{Study design and data collection}

This clinical observational study of children aged between one month to 15 years of age with a diagnosis of pancytopenia (hemoglobin $<10 \mathrm{~g} / \mathrm{dL}$, total leukocyte count $\leqslant 4 \times 10^{9} / \mathrm{L}$ and platelet count $\leqslant 100 \times 10^{9}$ ) [3] was conducted over one year from August 2015 to July 2016. The study was conducted by the Department of Pediatrics at the lead author's Medical University after clearance from the Institute Ethics Committee (Ref. PCM/II-B/P23 dated 29th October 2015). A written informed consent was obtained from patient's guardians in accordance with Helsinki 's declaration. Children receiving any form of antineoplastic chemotherapy or radiotherapy were excluded from the study.

Complete clinical history of all children including present and past illness, history of drug intake, family history of hematological disease, previous blood transfusions, dietary habits and jaundice along with the findings of the clinical examination were recorded. In all patients, a complete blood count was done. As per clinical condition and feasibility, other investigations like coagulation profile, liver function tests, kidney function tests, Vit B12 levels, blood folate levels, iron studies (serum iron, ferritin and total iron-binding capacity), bone marrow smears were made to detect aplasia/hypoplasia/megaloblasts/abnormal blasts, etc., and inclusion bodies of Parvovirus B19. When needed, additional diagnostic studies like HBsAg, antihepatitis C virus (HCV) antibody, Parvovirus B19 Immunoglobulin M (IgM), Immunoglobulin G (IgG); Epstein-Barr virus (EBV) IgM, IgG; cytomegalovirus (CMV) IgM, IgG antibodies, anti-HIV-1 \& HIV-2 antibody, gastric aspirate for acid-fast bacilli, chromosomal breakage studies were also performed.

\section{Statistical analysis}

Data were analyzed using the SPSS (Statistical Package for the Social Sciences, version 16.0, SPSS Inc, Chicago, IL, USA). Data were summarized in form of proportions and frequent tables for categorical variables. Continuous variables were summarized using means and standard deviation. Chi-square test was used for analyzing the association between various variables. A p-value of less than 0.05 was considered statistically significant.

\section{Results}

A total of 6,208 children were admitted to the Department of Pediatrics during the study period. Out of whom, 84 children (1.4\%) were diagnosed to have pancytopenia. Of these, 19 were female and 65 were male with a mean age at diagnosis of 70 months. Around $87 \%$ of children came from a rural background and the majority belonged to less affluent economic strata applying revised Kuppuswamy scales. Clinically, the majority of patients presented with complaints of fever (79\%), pallor (70\%) and history of previous blood transfusions (56\%); and almost half of the total (49\%) had episodes of bleeding like petechiae, ecchymoses and epistaxis (Table 1). 


\section{Cureus}

\begin{tabular}{|c|c|}
\hline Age in months: mean \pm SD & $70.77 \pm 4.8$ \\
\hline Sex & [n (\%)] \\
\hline Males & $65(77.4)$ \\
\hline Female & $19(22.6)$ \\
\hline Locality & [n (\%)] \\
\hline Rural & $73(86.9)$ \\
\hline Urban & $11(13.1)$ \\
\hline Socio-economic status & [n (\%)] \\
\hline Lower middle & $5(5.9)$ \\
\hline Upper lower & $47(55.9)$ \\
\hline Lower & $32(38.2)$ \\
\hline Clinical features & [n (\%)] \\
\hline Fever & $66(78.6)$ \\
\hline Pallor & $59(70.2)$ \\
\hline Previous blood transfusions & $47(56)$ \\
\hline Bleeding manifestations & $41(48.8)$ \\
\hline Hepatomegaly & $40(47.6)$ \\
\hline Splenomegaly & $27(32.1)$ \\
\hline Significant lymphadenopathy & $18(21.4)$ \\
\hline Pesticide exposure & $8(9.5)$ \\
\hline Tremors & $8(9.5)$ \\
\hline Jaundice & $4(4.8)$ \\
\hline Knuckle hyperpigmentation & $4(4.8)$ \\
\hline
\end{tabular}

TABLE 1: Clinical characteristics of study population $(n=84)$.

In order to assess the severity, the baseline hematological parameters were further classified according to the standard age and gender-matched reference ranges (Table 2). According to statistical analysis, no significant difference was found between the major diagnostic categories (megaloblastic anemia, aplastic anemia and acute lymphoblastic leukemia [ALL]) with regards to initial hematological parameters. 


\section{Cureus}

\begin{tabular}{|l|l|l|}
\hline Parameters & Frequency & $\%$ \\
\hline Moderate anemia & 7 & 8.3 \\
\hline Severe anemia & 77 & 91.7 \\
No neutropenia & 20 & 23.8 \\
Mild neutropenia & 24 & 28.6 \\
\hline Moderate neutropenia & 17 & 20.2 \\
\hline Severe neutropenia & 23 & 27.4 \\
\hline Lymphocytopenia present & 58 & 69 \\
Lymphocytopenia absent & 26 & 31 \\
PIt (<25,000) & 39 & 46.4 \\
Plt (25,000-50,000) & 19 & 22.6 \\
Plt (50,000-75,000) & 8 & 9.5 \\
PIt (75,000-100,000) & 18 & 21.5 \\
\hline
\end{tabular}

TABLE 2: Distribution of hematological parameters $(n=84)$.

Plt: platelets.

Bone marrow examination could be performed for definitive diagnosis of pancytopenia in 80 children and revealed aplastic changes in 37\%, hyperplastic in $14 \%$ and normal cellularity in $44 \%$ of them. The bone marrow findings in various etiology (Table 3) and outcome (Table 4) of pancytopenia found in our cohort are summarized below. 


\section{Cureus}

\begin{tabular}{|c|c|c|c|c|c|}
\hline \multirow[b]{2}{*}{ Diagnosis } & \multicolumn{4}{|c|}{ Bone marrow cellularity } & \multirow{2}{*}{ Total } \\
\hline & Aplastic & Normal & Hyperplastic & Not done & \\
\hline ALL & 1 & 12 & 6 & 0 & 19 \\
\hline AML & 1 & 2 & 0 & 0 & 3 \\
\hline Aplastic anemia & 27 & 0 & 0 & 0 & 27 \\
\hline Complicated malaria & 0 & 1 & 0 & 0 & 1 \\
\hline Hemolytic & 0 & 1 & 1 & 0 & 2 \\
\hline HIV & 0 & 2 & 0 & 0 & 2 \\
\hline Hemophagocytic syndrome & 0 & 1 & 0 & 0 & 1 \\
\hline ITP & 0 & 2 & 0 & 0 & 2 \\
\hline Kala azar & 0 & 1 & 0 & 0 & 1 \\
\hline Megaloblastic anemia & 0 & 5 & 4 & 0 & 9 \\
\hline IDA & 0 & 0 & 0 & 2 & 2 \\
\hline TB & 0 & 2 & 0 & 0 & 2 \\
\hline Thalassemia & 0 & 0 & 1 & 0 & 1 \\
\hline Hypersplenism & 0 & 1 & 0 & 0 & 1 \\
\hline Unclassified & 2 & 7 & 0 & 2 & 11 \\
\hline Total & 31 & 37 & 12 & 4 & 84 \\
\hline
\end{tabular}

TABLE 3: Bone marrow findings of study population $(n=84)$.

ALL: acute lymphoblastic leukemia; AML: acute myeloid leukemia; HIV: human immunodeficiency virus; ITP: immune thrombocytopenia; IDA: irondeficiency anemia; TB: tuberculosis. 


\section{Cureus}

\begin{tabular}{|c|c|c|c|c|c|}
\hline & & \multicolumn{3}{|l|}{ Outcomes } & \multirow{2}{*}{ Total } \\
\hline & & Survived & Not survived & Discontinued treatment & \\
\hline & Acute lymphoblastic leukemia & 13 & 1 & 5 & 19 \\
\hline & Acute myeloid leukemia & 2 & 1 & 0 & 3 \\
\hline & Aplastic anemia & 13 & 7 & 7 & 27 \\
\hline & Complicated malaria & 1 & 0 & 0 & 1 \\
\hline & Hemolytic & 2 & 0 & 0 & 2 \\
\hline & Hemophagocytic syndrome & 1 & 0 & 0 & 1 \\
\hline & HIV & 1 & 1 & 0 & 2 \\
\hline \multirow[t]{8}{*}{ Diagnosis } & IDA & 1 & 0 & 1 & 2 \\
\hline & ITP & 1 & 0 & 1 & 2 \\
\hline & Kala azar & 1 & 0 & 0 & 1 \\
\hline & Megaloblastic anemia & 8 & 0 & 1 & 9 \\
\hline & Tuberculosis & 2 & 0 & 0 & 2 \\
\hline & Thalassemia & 1 & 0 & 0 & 1 \\
\hline & Hypersplenism & 0 & 0 & 1 & 1 \\
\hline & Unclassified & 9 & 0 & 2 & 11 \\
\hline Total & & 56 & 10 & 18 & 84 \\
\hline
\end{tabular}

TABLE 4: Distribution of etiology and outcome in study population $(n=84)$.

HIV: human immunodeficiency virus; ITP: immune thrombocytopenia; IDA: iron-deficiency anemia.

Patients were followed till the end of the study period and their outcome was reported in form of survival, expiry or abandonment of treatment (absconded/left treatment against medical advice/took leave, etc.). A total of 67\% (56/84) pancytopenics survived, 12\% (10/84) expired and the rest discontinued treatment $(18 / 84)$ over one year. Aplastic anemia, acute leukemia significantly contributed to the mortality in our study (Table 4). The degree of neutropenia, lymphopenia, anemia and thrombocytopenia were not statistically associated with outcome. The severity of aplastic anemia was significantly associated with outcome; $\mathrm{p}=0.0278$ (Table 5).

\begin{tabular}{|c|c|c|c|c|}
\hline Severity of AA & Survived & Not survived & Discontinued treatment & Total \\
\hline Non-severe & 8 & 1 & 3 & 12 \\
\hline Severe & 4 & 1 & 3 & 8 \\
\hline Very severe & 1 & 5 & 1 & 7 \\
\hline
\end{tabular}

TABLE 5: Association of outcome with severity of aplastic anemia (AA).

$\mathrm{p}$-value $=0.0278$.

In the under-5 population, severe malnutrition was present in $25 \%$ pts [8/32 children had mid-upper arm circumference (MUAC) less than $11.5 \mathrm{~cm}$ ]. 38.4\% (20/52) of the patient population was underweight by BMI scoring in children above five years. Anthropometric parameters, MUAC \& BMI (body mass index), were significantly associated ( $\mathrm{p}$ values of 0.0171 and 0.0045 , respectively) with the outcome (Table 6 ). 


\section{Cureus}

\begin{tabular}{|c|c|c|c|c|}
\hline Parameters: (a)MUAC, (b)BMI & Survived & Not survived & Discontinued treatment & Total \\
\hline MUAC >11.5 cm & 19 & 1 & 4 & 24 \\
\hline$M U A C<11.5 \mathrm{~cm}$ & 2 & 2 & 4 & 8 \\
\hline Underweight by BMI & 10 & 5 & 5 & 20 \\
\hline Normal BMI & 24 & 1 & 2 & 27 \\
\hline Overweight by BMI & 1 & 1 & 3 & 5 \\
\hline
\end{tabular}

TABLE 6: Association of outcome with anthropometrical parameters.

(a) MUAC (mid-upper arm circumference), p-value $=0.0171$ (chi-square test).

(b) BMI (body mass index), p-value $=0.0045$ (chi-square test).

\section{Discussion}

In the present observational, hospital-based study, the incidence of pancytopenia was found to be $1.4 \%$ with a male to female ratio of $3.4: 1$ and $\sim 85 \%$ population belonging to rural setting similar to other studies done in India [4-6]. Referral of rural population to our tertiary care center with preference to male children for health issues and less health awareness might explain the sex disparity.

We are aware that bone marrow aspiration and biopsy evaluation is of utmost importance to evaluate the causes of new-onset pancytopenia and plan further investigations $[1,2,7]$. In the present study, bone marrow examination was performed for 80 out of 84 patients as a pancytopenic infant of two months expired within three days and three patients refused for bone marrow. Out of 31 bone marrows that were found to be acellular/aplastic, two patients were labelled as transient cytopenia (probably viral in origin; as myelosuppression is accompanied by EBV and CMV positivity) wherein the counts recovered after seven to 10 days. The patients were asked to remain in follow-up. Here, despite an aplastic marrow a firm diagnosis of aplastic anemia couldn't be made. As substantiated by Weinzierl who emphasized that a significant fraction of cases in both children and adults demonstrated nonspecific marrow findings that required clinical follow-up and/or repeat biopsy for definitive diagnosis in a detailed review published a few years back [1].

Data obtained for relative frequency of different causes of pancytopenia was compared with other studies in and around India. Aplastic anemia was the most common cause accounting for 27 cases (32.1\%) in this study. Comparing with other studies, a similar incidence to our study findings was demonstrated by Naseem et al [8]. Savage et al [9] also reported it to be the second most common cause (26.1\%). Kumar et al [3] found it to be the commonest cause (29.5\%) of pancytopenia among adults at a hematology center, due to the high proportion of referred cases at their center. As a hematological disorder with severe dyscrasias, aplastic anemia is more prevalent in Asia compared to the West. European studies indicate the annual incidence of only two new cases per million of population [10]. However, studies done in China [11] and Thailand [12] reported that the frequency of aplastic anemia is three folds greater than that of the western countries of the world. The age of aplastic anemia patients varied from two to 14 years with a mean age of 8.33 years in the present study which is similar to the results of Khodke et al [4]. Sex distribution of aplastic anemia in our study was found to be 3.5:1 (males: females) as opposed to 1:1 of Turkey [13] and 1.06 in a French study [14]. This male preponderance in the demographic profile is due to preferential reporting of the disease from the male counterparts of the society, especially from the rural areas, which constitutes $77.7 \%$ of the study group of aplastic anemia. No etiologic factor could be implicated in the majority of the cases in the present study with aplastic anemia similar to findings by Mishra et al [15].

The second important cause in our study was leukemia diagnosed in $26.2 \%$ (22/84) patients comparable to $17.4 \%$ incidence as reported by Rathod et al [16]. Amidst our 22 patients, only three had acute myeloid leukemia while the rest 19 belonged to acute lymphoblastic leukemia; the most common type of childhood malignancy. In the present study, mean age of leukemia patients was found to be 5.5 years (range 2-11 years). Morphological breakup of leukemia population reveals normocytic picture in 10 (nine with increased red cell distribution width [RDW] whereas in one RDW was not sent initially) microcytic in eight and macrocytic in four patients. The picture may have been confounded by haematinics, prior transfusions and iron, folate \& Vit B12 deficiency, etc.

The third most common cause was found to be nutritional anemia with an incidence of $13.1 \%$ comparable to the findings of Naseem et al [8] and Kumar et al [17]. Another interesting observation in the present study was that vitamin B12 deficiency was found to be more common than folate deficiency in patients with 
megaloblastic anemia -consistent with the results of similar studies conducted in India and around $[18,19]$. Vit B12 levels and folate levels could be done in 46 patients only due to affordability issues and 16 of them (35\%) had deficient B12 levels- present in seven patients with nutritional anemia, five patients of acute leukemia, two aplastic anemia, one with transient cytopenia and one with miliary TB. Folate deficiency was reported in 3/46 patients - one with thalassemia, miliary TB and a patient with leukemia. The miscellaneous group comprised of infections and hematological disorders like malaria, TB, hemophagocytic

lymphohistiocytosis (HLH), thalassemia, unclassified, etc. Results came positive for Parvovirus B19 in 8\%, EBV in $7 \%$ CMV in $1 \%$, Hepatitis in $2.4 \%$, gastric aspirate found acid-fast bacilli in $\sim 4 \%$ and paroxysmal nocturnal hemoglobinuria $(\mathrm{PNH})$ clones were detected in $~ 5 \%$ cases. Most patients in this group had counts recovery within 10 days; probably transient (secondary to viral; could not be assayed). So, it is implied that even benign causes might present with severe pancytopenia if symptoms are neglected for long or diagnosis is delayed.

This observational study also aimed to study factors associated with poor outcome in pancytopenic patients. Patient's outcome depends on the etiology of pancytopenia, treatment modalities, supportive care, and the era in which results are analyzed. This is exemplified by advancements in the management of aplastic anemia over the decades. In a large series conducted before 1957, Wolff [20] found a survival rate of only $3 \%$ in aplastic anemia whereas in a 2004 series [21], 12\% had a spontaneous resolution, $21 \%$ remained in the moderate range and $67 \%$ progressed to severe aplastic anemia. In our study, the mortality was found to be highest in the age group of five to 10 years in which aplastic anemia and acute leukemia were the causative factors. Seven out of 10 expired children were boys but should not give a false impression of more vulnerability in males as there is no equal representation of both sexes in the study population (male:female $=3.4: 1$ in our study population). Notably, the proportion of deaths in males is $11 \%(7 / 65)$ which is less than that in females at $15.7 \%(3 / 19)$. Socio-cultural perspective of preferential presentation for treatment in male children and tendency for discontinuing treatment in female children by guardians especially hesitation and reluctance for arranging blood for advised transfusions can explain the reported data.

The etiology-wise morbidity and mortality distribution highlights some interesting observations (Table 3). The highest contributor to mortality in our data series was made by aplastic anemia, it was seen that despite the same protocol of immunosuppressive therapy (ATG along with cyclosporine) and hospital-based transfusions to all the 27 aplastic anemia pts in our study, outcome differed across groups. This was seen to depend on the severity of aplastic anemia ( $p$-value $=0.0278$; Table 5), sepsis control along with prophylactic and therapeutic blood transfusions. The patients with very severe aplastic anemia had the worst outcome with only one of the seven patients surviving despite upgraded antibiotics, blood transfusions (Table 5). The confounding reasons could have been poor pre-morbid state, severe infections considering the low blood counts and more need for transfusions before admission. Kulkarni (2015) et al [22] asserted that the clinical presentation of patients with pancytopenia clearly differed from the ALL patients without pancytopenia at presentation. These clinical indicators may denote less aggressive nature of the ALL blasts and of the disease in these patients and may potentially point to a distinct biology of disease in them [22]. This was also seen in our study with only two out of 22 leukemia patients not surviving (one expired after the beginning of induction chemotherapy). Hence, $70 \%$ of the total deaths occurred in patients with aplastic anemia, 20\% in leukemia and benign diseases like megaloblastic anemia, hemolytic anemia and infections like kala azar, etc., did not add to morbidity.

Patients younger than five years old with severe wasting and smaller MUAC had a poor outcome (Table 6) so did underweight pancytopenic children above five years; contributed by etiology, pre-morbid state and infections. We found that the negative outcome of children is significantly associated with low anthropometrical values ( $\mathrm{p}$-value $<0.05$ ). All these factors were related to nutritional status of child and finally to overall immunity and capability of him/her to cope up with infections, transfusion-related complications and severe stage of anemia. Though not found to be statistically significant ( $\mathrm{p}$-value $>0.05$ ) but severe anemia was observed in all the 10 expired patients. Also lymphocytopenia (seen against standard age-specific reference ranges) was present in $70 \%$ of the expired patients. Similarly, $40 \%$ of the expired patients had severe neutropenia. Obviously, these isolated findings should not be extrapolated as the outcome is an amalgamation of multiple factors.

The present study adds to socio-demographic and clinical profile of the varied spectrum of etiology and severity of pancytopenia in children, of which potentially treatable and reversible miscellaneous causes like infections and nutritional deficiencies emerged as a causative factor in a notable percentage of the pancytopenia cases. However, the majority was accounted for by the acute leukemia and aplastic anemia at our referral tertiary care center. Detailed hematological profiles of the entities were traced but failed to show any marked difference at initial presentation. Emerging use of estimation of serum vitamin B12, folate levels, viral markers, PNH clones and parasitological tests along with the use of invasive procedures like bone marrow aspiration aid in evaluating cytopenia and in its definitive management. Low counts increase morbidity and recovery rates become poor getting further clouded by the need for recurrent transfusions as well as active sepsis control. Notable socio-cultural perspective in a developing country like India in form of socio-demographic variables like gender, locality (urban vs rural) tend to have a significant effect as they play a role in a timely presentation to health service, causative exposure (to pesticides), replacement blood donation and general health condition. Cultural factors like denial for replacement blood transfusion [23], nutritional anemia and worm infestations might skew the expected clinical findings as seen in the present 
study.

The present study has limitations in form of limited patient population (84), follow-up period (one year) and logistic issues (unaffordability for some investigations). As already stated, the study setting was in a tertiary care center located in the capital of a densely populated state getting referrals from north India, so skewness in form of a higher incidence of malignant causes may be expected. A more detailed study with a greater duration of follow-up may outline the course of disease and effects of each of these variables on final outcome.

\section{Conclusions}

The data gathered support a complex picture for pancytopenia in our study population since both benign nutritional deficiencies and malignant hematological neoplasms were noted. A good clinical correlation is of utmost importance to evaluate each specific case and plan for further hematological evaluation and appropriate management as the outcome is multifactorial.

\section{Additional Information}

\section{Disclosures}

Human subjects: Consent was obtained or waived by all participants in this study. Institutional Ethics Committee, King George's Medical University issued approval PCM/II-B/P23 dated 29th October 2015. Institutional Ethics Committee, King George's Medical University approved the work ref PCM/II-B/P23 dated 29th October 2015. Animal subjects: All authors have confirmed that this study did not involve animal subjects or tissue. Conflicts of interest: In compliance with the ICMJE uniform disclosure form, all authors declare the following: Payment/services info: All authors have declared that no financial support was received from any organization for the submitted work. Financial relationships: All authors have declared that they have no financial relationships at present or within the previous three years with any organizations that might have an interest in the submitted work. Other relationships: All authors have declared that there are no other relationships or activities that could appear to have influenced the submitted work.

\section{Acknowledgements}

Prof Farzana Alim, MSc PhD, AMU, Aligarh, India with specialisation in child welfare and psychology for helping in the evaluation of socio-cultural aspects of this problem in pediatric population in North India. Prof Abdul Alim, M Phil, PhD, AMU, Aligarh, India for his expertise in languages and teaching experience in literature for helping in proofreading the work.

\section{References}

1. Weinzierl EP, Arber DA: Bone marrow evaluation in new-onset pancytopenia . Hum Pathol. 2013, 44:115464. 10.1016/i.humpath.2012.10.006

2. Jain A, Naniwadekar M: An etiological reappraisal of pancytopenia - largest series reported to date from a single tertiary care teaching hospital. BMC Hematol. 2013, 13:10. 10.1186/2052-1839-13-10

3. Kumar R, Kalra SP, Kumar H, Anand AC, Madan H: Pancytopenia--a six year study. J Assoc Physicians India. 2001, 49:1078-81.

4. Khodke K, Marwah S, Buxi G, Vadav RB, Chaturvedi NK: Bone marrow examination in cases of pancytopenia. J Academy Clin Med. 2001, 2:55-9.

5. Tilak V, Jain R: Pancytopenia--a clinico-hematologic analysis of 77 cases . Indian J Pathol Microbiol. 1999, 42:399-404.

6. Dubey SR, Patel SK, Arya AK, Singh RP: Clinico-etiological spectrum of pancytopenia in hospitalized children. Int J Contemp Pediatr. 2016, 3:169-72. 10.18203/2349-3291.ijcp20160153

7. Vaidya S: Evaluation of bone marrow in cases of pancytopenia in a tertiary care hospital . J Pathol Nepal. 2015, 5:691-5. 10.3126/jpn.v5i9.13686

8. Naseem S, Varma N, Das R, Ahluwalia J, Sachdeva MU, Marwaha RK: Pediatric patients with bicytopenia/pancytopenia: review of etiologies and clinico-hematological profile at a tertiary center. Indian J Pathol Microbiol. 2011, 54:75-80. 10.4103/0377-4929.77329

9. Savage DG, Allen RH, Gangaidzo IT, et al.: Pancytopenia in Zimbabwe. Am J Med Sci. 1999, 317:22-3. 10.1097/00000441-199901000-00004

10. Young NS, Calado RT, Scheinberg P: Current concepts in the pathophysiology and treatment of aplastic anemia. Blood. 2006, 108:2509-19. 10.1182/blood-2006-03-010777

11. Yang C, Zhang X: Incidence survey of aplastic anemia in China . Chin Med Sci J. 1991, 6:203-7.

12. Issaragrisil S, Kaufman DW, Anderson T, Chansung K, Leaverton PE, Shapiro S, Young NS: The epidemiology of aplastic anemia in Thailand. Blood. 2006, 107:1299-307. 10.1182/blood-2005-01-0161

13. Başlar Z, Aktuğlu G, Bolaman Z, et al.: Incidence of aplastic anemia in Turkey: a hospital-based prospective multicentre study. Leukemia Res. 1997, 21:1135-9. 10.1016/s0145-2126(97)00046-5

14. Mary JY, Baumelou E, Guiguet M: The French Cooperative Group for Epidemiological Study of Aplastic Anemia. Epidemiology of Aplastic Anemia in France: a prospective multicentric study. Blood. 1990, 75:164653.

15. Mishra D, Kohli A, Yadav R, Chandra S: Pancytopenia in Indian children: a clinico-hematological analysis . Indian J Clin Pract. 2018, 28:759-62. 


\section{Cureus}

16. Rathod GB, Alwani M, Patel H, Jain A: Clinicohematological analysis of pancytopenia in pediatric patients of tertiary care hospital. IAIM. 2015, 2:15-9.

17. Kumar D, Raghupathi A: Clinicohematologic analysis of pancytopenia: study in a tertiary care centre . Basic Appl Pathol. 2012, 5:19-21. 10.1111/j.1755-9294.2011.01121.x

18. Premkumar M, Gupta N, Singh T, Velpandian T: Cobalamin and folic acid status in relation to the etiopathogenesis of pancytopenia in adults at a tertiary care centre in north India. Anemia. 2012, 2012:707402. 10.1155/2012/707402

19. Iqbal SP, Kakepoto GN, Iqbal SP: Vitamin B12 deficiency--a major cause of megaloblastic anaemia in patients attending a tertiary care hospital. J Ayub Med Coll Abbottabad. 2009, 21:92-4.

20. Wolff JA: Anemias caused by infections and toxins, idiopathic aplastic anemia and anemia caused by renal disease. Pediatr Clin North Am. 1957, 4:469-80. 10.1016/s0031-3955(16)30512-0

21. Howard SC, Naidu PE, Hu XJ, Jeng MR, Rodriguez-Galindo C, Rieman MD, Wang WC: Natural history of moderate aplastic anemia in children. Pediatr Blood Cancer. 2004, 43:545-51. 10.1002/pbc.20131

22. Kulkarni KP, Marwaha RK: Acute lymphoblastic leukemia with pancytopenia at presentation: clinical correlates, prognostic impact, and association with survival. J Pediatr Hematol Oncol. 2013, 35:573-6. 10.1097/MPH.0b013e31829d46f3

23. Alim M, Abdul Rahman R, Goel P: Replacement blood donation denials in children: a cross-sectional study . I Clin Diagn Res. 2021, 15:5-8. 10.7860/TCDR/2021/48990.14865 